\title{
Lozenge tilings with free boundary
}

\author{
Greta Panova $\|^{\prime \prime}$ \\ ${ }^{1}$ University of Pennsylvania, Philadelphia, PA 19102, USA
}

\begin{abstract}
We study tilings with lozenges of a domain with free boundary conditions on one side. These correspond to boxed symmetric plane partitions. We show that the positions of the horizontal lozenges near the left flat boundary, in the limit, have the same joint distribution as the eigenvalues from a Gaussian Unitary Ensemble (the GUE-corners/minors process). We also prove the existence of a limit shape of the height function (the symmetric plane partition). We also consider domains where the sides converge to $\infty$ at different rates and recover again the GUE-corners process.
\end{abstract}

Résumé. Nous étudions les pavages par losanges d'un domaine dont le bord vertical est "libre". Nous montrons que les positions des losanges horizontaux proches du bord gauche ont la même distribution que les valeurs propres de l'ensemble gaussien unitaire. Nous montrons aussi l'existence d'une limite de la forme de la fonction de hauteur (une partition plane symétrique). Nous considérons aussi des domaines ou des bords différents convergent vers $\infty$ des taux différents et nous retrouvons nouveau les processus EGU au bord.

Keywords: lozenge tilings, symmetric plane partitions, limit shapes, GUE eigenvalues

\section{Introduction}

We study lozenge tilings of certain domains with partially free boundary conditions. These are tilings of a domain on a triangular grid into unit-sided lozenges, see Figure 1. In the particular setting, we are interested in a half-hexagonal domain, such that on its side corresponding to the main diagonal of the hexagon, we have free boundary conditions - that is, the "horizontal" lozenges are allowed to cross that (free) boundary at any place and protrude halfway through. Let $m$ be the length of the vertical side of the hexagon, let $n$ the other two lengths, and denote by $T_{f}(n, m)$ the set of all so-described free-boundary tilings. Reflecting the tiling along the right boundary line of the domain gives a symmetric tiling, which corresponds to a boxed symmetric plane partition fitting in an $m \times n \times n$ box (each lozenge represents a side of a cube).

Lozenge tilings of fixed boundary domains have been studied extensively both as combinatorial objects corresponding to plane partitions (see e.g. [EC2]) and as integrable models in statistical mechanics, where the interest has been the limiting behavior as the mesh (triangle) size goes to 0 and the domain has a fixed boundary. In these cases, most aspects of the limit behavior have been understood - the "frozen regions" (covered by just one type of tile) bounded by algebraic "arctic curves" (see e.g. [KO], [CLP]), the surface

\footnotetext{
†Email: panova@math. upenn.edu

1365-8050 @ 2015 Discrete Mathematics and Theoretical Computer Science (DMTCS), Nancy, France
} 
as a limit of the height function, also referred to as "limit shape", (see [BBO, BG, CLP, CKP]), the fluctuations near the frozen boundary being the Airy process (see [Petrov] and references therein) and the fact that the positions of the horizontal lozenges near a flat vertical boundary have the same joint distribution as the eigenvalues of Gaussian Unitary Ensemble (GUE) matrices (see [GP, OR] and works by [Johansson-Nordenstam],[Nordenstam], [Novak]). Symmetric lozenge tilings (plane partitions) have also been studied when the symmetry is along the horizontal axis instead of the vertical axis as considered here (referring to Figure 1 , with results by [Forrester-Nordenstam] and in [BG].

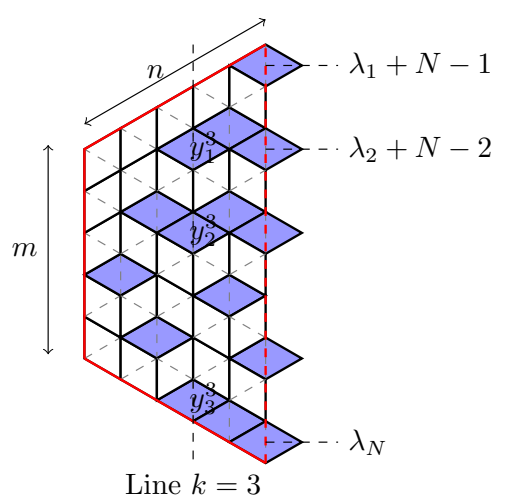

Line $k=3$
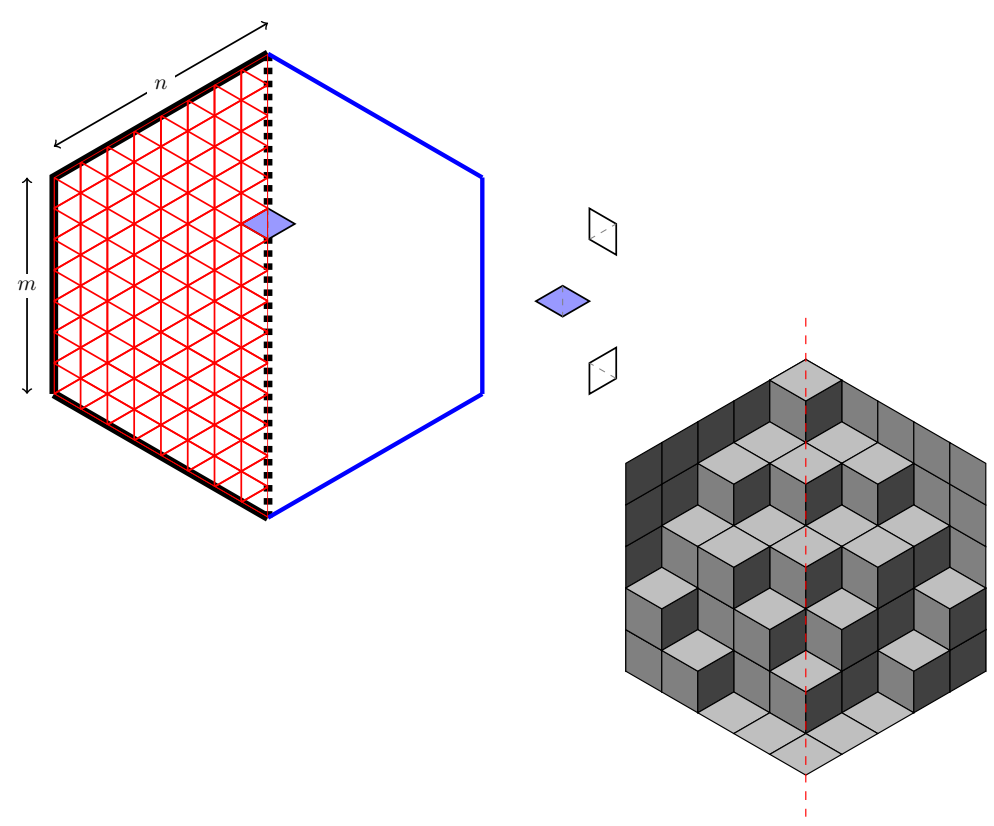

Fig. 1: Top, middle: the half-hexagon domain in the triangular grid, which is tiled with the 3 types of lozenges (on the right) - the right boundary of the domain (middle dashed line) is allowed to cut a lozenge in half as shown and the positions of these "cut" lozenges are unrestricted. Left: the positions $y^{k}$ of the horizontal lozenges on vertical line $k$ of a tiling whose rightmost boundary is given by the partition $\lambda=(5,4,3,1,0)$, here $k=3$ and $y^{3}=(4,3,0)$. Right: the corresponding vertically-symmetric boxed plane partition.

Here we study the same aspects for lozenge tilings with free boundaries as the ones studied for fixed domains, described above. We show that the positions of the horizontal lozenges near the left boundary have the same distribution as the GUE matrices eigenvalues, see Theorem 4.3 We also prove the existence of the limit shape of the height function (i.e. the symmetric plane partition) in Theorem 5.3. The existence of the limit shape was assumed (without proof) in $[\mathrm{DR}]$, where the limit shape and arctic curves were calculated under these assumptions, but the limit shape existence in such tilings has not been studied with the present variational methods.

Theorem 1.1 Let $m / n \rightarrow a$ as $n, m \rightarrow \infty$. The rescaled height function of the uniformly random lozenge tilings of a half-hexagon with free right boundary (i.e. the symmetric plane partition) converges 
(in probability) to a unique limit shape, which coincides over the half-hexagon with the limit shape for the tilings of the full hexagon (fixed boundary) as described in $[C L P]$. Moreover, the positions of the horizontal lozenges (shifted by $m / 2$ and rescaled by $\sqrt{n\left(a^{2}+2 a\right) / 8}$ ) on the $r$-th vertical line from the left have the same joint distributions as $n, m \rightarrow \infty$, which is the distribution of the eigenvalues of $r \times r$ matrices from the Gaussian Unitary Ensemble. Further, the joint distribution of these positions on all lines $1, \ldots, k$ converges to the joint distribution of the collection of the eigenvalues of the principal submatrices of sizes $1, \ldots, k$ of a GUE matrix, known as the GUE-corners (GUE-minors) process.

Our methods to proving the GUE distribution follow the approach developed in [GP] on asymptotics of symmetric functions through certain integral representations and steepest descent asymptotic analysis. There GUE was shown for tilings of fixed boundary domains by using asymptotics of their moment generating functions, which were certain Schur functions. As it turns out here, the role of Schur functions is replaced by symplectic characters and so we derive their asymptotics explicitly. The proof of the existence of the limit shape follows the approach of $[\overline{\mathrm{BBO}}]$ and $[\mathrm{BG}]$ with moment generating functions for certain measures. It relies on some further symmetric function identities and their asymptotics. Finally, the GUE phenomenon for differently scaled $m$ and $n$ is also shown through asymptotic analysis of symplectic characters using the exact formulas from [GP] with care on the analysis in the unusual regimes. For the complete proofs, technical definitions and statements, and the full list of references we refer the reader to the full version of this paper, available at ARXIV:1408.0417.

\section{Preliminaries}

Rational irreducible representations of Lie groups are parametrized by their highest weights (also called signatures or integer partitions when the entries are nonnegative integers) $\lambda$-sequences of (half)integers $\lambda=\left(\lambda_{1} \geq \lambda_{2} \geq \cdots \geq \lambda_{N}\right)$. Denote the set of highest weights/signatures of length $N$ by $G T_{N}$. We will use the characters of these representations. For $G L_{N}(\mathbb{C})$, these are the Schur functions $s_{\lambda}\left(x_{1}, \ldots, x_{N}\right)$, which can be defined for any sequence $\lambda$ via Weyl's character formula, and when $\lambda$ is an integer partition these are the generating functions for semi-standard Young tableaux of shape $\lambda$, see [EC2, Mac]. The value of the character of the irreducible representation of the symplectic group $S p_{2 N}(\mathbb{C})$, parameterized by $\lambda \in G T_{N}$, on a symplectic matrix with eigenvalues $x_{1}, x_{1}^{-1}, \ldots, x_{N}, x_{N}^{-1}$ is given by

$$
\chi_{\lambda}\left(x_{1}, \ldots, x_{N}\right)=\frac{\operatorname{det}\left[x_{i}^{\lambda_{j}+N+1-j}-x_{i}^{-\left(\lambda_{j}+N+1-j\right)}\right]_{i, j=1}^{N}}{\operatorname{det}\left[x_{i}^{N+1-j}-x_{i}^{-N-1+j}\right]_{i, j=1}^{N}} .
$$

We now repeat definitions, results and setups from [GP] which will be used later. The normalized Schur function/ symplectic character on $k$ variables are defined as

$$
S_{\lambda}\left(x_{1}, \ldots, x_{k} ; N\right)=\frac{s_{\lambda}\left(x_{1}, \ldots, x_{k}, 1^{N-k}\right)}{s_{\lambda}\left(1^{N}\right)} \quad \mathfrak{X}_{\lambda}\left(x_{1}, \ldots, x_{k} ; N\right)=\frac{\chi_{\lambda}\left(x_{1}, \ldots, x_{k}, 1^{N-k}\right)}{\chi_{\lambda}\left(1^{N}\right)} .
$$

Proposition 2.1 (Proposition 3.19 in [GP]) For any signature $\lambda \in \mathbb{G T}_{N}^{+}$we have

$$
\mathfrak{X}_{\lambda}(x ; N)=\frac{2}{x+1} S_{\nu}(x ; 2 N, 1),
$$

where $\nu \in \mathbb{G T}_{2 N}$ is given by $\nu_{i}=\lambda_{i}+1$ for $i=1, \ldots, N$ and $\nu_{i}=-\lambda_{2 N-i+1}$ for $i=N+1, \ldots, 2 N$. 
In [GP] explicit asymptotic expansions for the normalized Schur functions $S_{\lambda}$ were found, when $\lambda(N)$ converges to a limiting profile function $f$ on $[0,1]$ in the sense of $\lambda(N)_{i} / N \rightarrow f(i / N)$ under specific conditions as $N \rightarrow \infty$ for $i=1, \ldots, N$. The precise results and convergence conditions can be found in $[\mathrm{GP}]$ and the full version of this paper, here we just state them informally.

Proposition 2.2 (Proposition 4.3 in [GP]) Suppose that $f(t)$ is piecewise-differentiable, and $\lambda(N) / N \rightarrow f$ as $N \rightarrow \infty$ in a certain sense (see $[G P])$. Then for any fixed $h \in \mathbb{R}$

$$
\begin{gathered}
S_{\lambda(N)}\left(e^{h / \sqrt{N}} ; N\right)=\exp \left(\sqrt{N} E(f) h+\frac{1}{2} S(f) h^{2}+o(1)\right) \quad \text { as } N \rightarrow \infty, \text { where } \\
E(f)=\int_{0}^{1} f(t) d t, \quad S(f)=\int_{0}^{1} f(t)^{2} d t-E(f)^{2}+\int_{0}^{1} f(t)(1-2 t) d t .
\end{gathered}
$$

Moreover, the error o(1) is uniform over h belonging to compact subsets of $\mathbb{R} \backslash 0$.

Proposition 2.3 (Proposition 4.1 in $[\mathbf{G P}]$.) For $y \in \mathbb{R} \backslash\{0\}$, suppose that $f(t)$ is piecewise-continuous, $\lambda(N) / N \rightarrow f$ in a certain sense, and $w_{0}=w_{0}(y)$ is the (unique) real root of $(\partial / \partial w) \mathcal{F}(w ; y)=0$, where $\mathcal{F}(w ; f)=\int_{0}^{1} \ln (w-(f(t)+1-t)) d t$. If $y \in \mathbb{R} \backslash\{0\}$ id such that $w_{0}$ is outside the interval $\left[\frac{\lambda_{N}(N)}{N}, \frac{\lambda_{1}(N)}{N}+1\right]$ for all $N$ large enough, then

$$
\lim _{N \rightarrow \infty} \frac{\ln S_{\lambda(N)}\left(e^{y} ; N\right)}{N}=y w_{0}-\mathcal{F}\left(w_{0}\right)-1-\ln \left(e^{y}-1\right) .
$$

We use the formula for the multivariate symplectic characters from [GP, Theorem 3.17] to derive the asymptotics for $k>1$ out of developed asymptotics for $k=1$ :

$$
\begin{aligned}
\mathfrak{X}_{\lambda}\left(x_{1}, \ldots, x_{k} ; N\right) & =\frac{\Delta_{s}^{1}\left(1^{N}\right)}{\Delta_{s}^{1}\left(x_{1}, \ldots, x_{k}, 1^{N-k}\right)} \times \\
(-1)^{\left(\begin{array}{c}
k \\
2
\end{array}\right)} \operatorname{det}\left[\left(x_{i} \frac{\partial}{\partial x_{i}}\right)^{2(j-1)}\right]_{i, j=1}^{k} & \prod_{i=1}^{k} \mathfrak{X}_{\lambda}\left(x_{i} ; N\right) \frac{\left(x_{i}-x_{i}^{-1}\right)\left(2-x_{i}-x_{i}^{-1}\right)^{N-1}}{2(2 N-1) !},
\end{aligned}
$$

where the $\Delta_{s}^{1}$ are Vandermonde-type explicit polynomials.

We use the following crucial formula from [Mac, I.5, Example 16], which we refer to as Macdonald's identity, to compute the sum of Schur functions, indexed by partitions $\lambda \subset\left(\mathrm{m}^{n}\right)$ :

$$
\phi_{m}\left(x_{1}, \ldots, x_{n}\right):=\sum_{\lambda \in\left(m^{n}\right)} s_{\lambda}\left(x_{1}, \ldots, x_{n}\right)=\frac{\operatorname{det}\left[x_{j}^{m+2 n-i}-x_{j}^{i-1}\right]_{1 \leq i, j \leq n}}{\operatorname{det}\left[x_{j}^{2 n-i}-x_{j}^{i-1}\right]_{1 \leq i, j \leq n}} .
$$

The right-hand side of (4) is also (a shifted version of) Weyl's dimensional formula for the character $\gamma_{\lambda}$ corresponding to the irreducible representation of highest weight $\lambda=(\mathrm{m} / 2)$ of the odd orthogonal group $O_{2 n+1}(\mathbb{C})$.Using a combinatorial interpretation by Seshadri coming from algebraic geometry, Macdonald's identity (4) is evident in Proctor's work and further generalized by Krattenthaler. It relies on the "branching rule" for the restriction of a representation of $o(2 n+1)$ to a subalgebra $\operatorname{sl}(n)$. However, no direct combinatorial proof is known relating the semi-standard Young tableaux of $n$ letters which fit into 
the $\left(m^{n}\right)$ rectangle, and the combinatorial interpretation of the orthogonal characters, coming from the $S_{2 n-1} \hookrightarrow S_{2 n+1}$ branching rule, which corresponds to certain "half" Gelfand-Tsetlin triangles.

In order to take advantage of some established relationships between symplectic characters and Schur functions from [GP], we express (using Weyl's character formula), $\phi_{m}$ as a ratio of symplectic characters. Denote $\tau^{r}=\left((r / 2-1 / 2)^{n}\right)$. After certain algebraic manipulations we derive the special moment generating function (m.g.f.) for tilings from $T_{f}(n, m)$, whose role will become evident later:

$$
\Phi_{m}\left(x_{1}, \ldots, x_{k} ; n\right)=\frac{\phi_{m}\left(x_{1}, \ldots, x_{k}, 1^{n-k}\right)}{\phi_{m}\left(1^{n}\right)}=\prod_{i=1}^{k} x_{i}^{\frac{m}{2}} \frac{\mathfrak{X}_{\tau^{m}}\left(x_{1}, \ldots, x_{k} ; n\right)}{\mathfrak{X}_{\tau^{0}}\left(x_{1}, \ldots, x_{k} ; n\right)} .
$$

\section{Asymptotics I: when $\lim _{n \rightarrow \infty} \frac{m}{n} \in(0,+\infty)$}

We derive the asymptotic behavior for the normalized symplectic characters and so via $(\diamond)$ for $\Phi$.

Proposition 3.1 When $\frac{m}{n} \rightarrow$ a for $0<a<\infty$ we have that

$$
\mathfrak{X}_{\tau^{m}}\left(e^{\frac{h}{\sqrt{n}}} ; n\right)=\exp \left(\frac{1}{4}\left(a^{2}+a\right) h^{2}+o(1)\right) \quad \text { and } \quad \mathfrak{X}_{\tau^{0}}\left(e^{h / \sqrt{n}} ; n\right)=1+o(1),
$$

where the error terms o(1) converge to 0 uniformly on compact real domains for $h$.

Proof sketch: Using Proposition 2.1 we derive asymptotic formulas for $S_{\nu^{m}}\left(e^{\frac{h}{\sqrt{2 n}}} ; 2 n, 1\right)$ when $m / n \rightarrow$ $a \neq 0, \infty$ and also when $m=0$. In the $m \neq 0$ case we apply the formula 2.2 directly, in the $m=0$ we use the integral formula for the normalized Schur functions from [GP, Section 3.2] to derive that $S_{\lambda}(x ; N)=S_{\widehat{\lambda}}\left(x^{1 / \beta} ; N\right)$ for $\widehat{\lambda}_{i}=\beta \lambda_{i}+(\beta-1)(N-i)$ and then apply the same analysis with $\widehat{\nu^{0}}$ which now satisfies the conditions in Proposition 2.2 .

Proposition 3.2 Let $\left\{a_{i}\right\}_{i=1}^{\infty},\left\{b_{i}\right\}_{i=1}^{\infty}$ be sequences of positive real numbers, such that $a_{N} \rightarrow 0$ and $\frac{b_{N}}{N} \rightarrow 0$ as $N \rightarrow \infty$. Suppose that for some number $b$ we have $\mathfrak{X}_{\lambda(N)}\left(e^{a_{N} y} ; N\right) e^{b_{N} y} \rightarrow g(y)$ uniformly on compact subsets of a domain $D \subset \mathbb{C}$ as $N \rightarrow \infty$. Then

$$
\lim _{N \rightarrow \infty} \mathfrak{X}_{\lambda(N)}\left(e^{y_{1} a_{N}}, \ldots, e^{y_{k} a_{N}} ; N\right) e^{b_{N}\left(y_{1}+\cdots+y_{k}\right)}=g\left(y_{1}\right) \cdots g\left(y_{k}\right)
$$

uniformly on compact subsets of $D^{k}$.

This result will be used primarily for $a_{N}=1 / \sqrt{N}$ and $b_{N}=0$ in the case when $m / n$ is bounded nonzero, and later in Section 6 with $a_{n}=\frac{\sqrt{n}}{m}$ and $b_{n}=0$ when $m / n \rightarrow \infty$. The proof uses formula (3) with certain substitutions and bounds on the derivatives, and will be omitted in this abstract.

Proposition 3.3 Suppose that $\lambda(N)$ is a sequence of signatures, such that $\frac{\ln \mathfrak{X}_{\lambda(N)}(u ; N)}{N} \rightarrow$ $X(u)$, as $N \rightarrow \infty$, where the convergence is uniform in a complex neighborhood of $u=1$. Then

$$
\frac{\ln \mathfrak{X}_{\lambda(N)}\left(u_{1}, \ldots, u_{k} ; N\right)}{N} \rightarrow X\left(u_{1}\right)+\cdots+X\left(u_{k}\right)
$$

as $N \rightarrow \infty$, where the convergence is uniform in a neighborhood of $\left(1^{k}\right)$ in $\mathbb{C}^{k}$. 
Proof sketch: The proof uses the observation that $\frac{\left(\frac{\partial}{\partial u}\right)^{j} \mathfrak{X}_{\lambda}(u ; N)}{\mathfrak{X}_{\lambda}(u ; N)} \in$ $\mathbb{Z}\left[\frac{\partial}{\partial u} \ln \mathfrak{X}_{\lambda}(u ; N), \ldots, \frac{\partial^{j}}{\partial u^{j}} \ln \mathfrak{X}_{\lambda}(u ; N)\right]$, i.e. it lhs is a degree $j$ polynomial in the given ring. This allows us to apply formula (3) with suitable substitutions/normalizations.

Applying the asymptotic results above together with equation $(\diamond)$ gives the following.

Theorem 3.4 For any fixed $y_{1}, y_{2}, \ldots, y_{k} \in \mathbb{R}$ we have that

$$
\Phi_{m}\left(e^{y_{1} / \sqrt{n}}, \ldots, e^{y_{k} / \sqrt{n}} ; n\right) \exp \left(-\left(\frac{m}{2 \sqrt{n}}\right) \sum_{i=1}^{k} y_{i}\right) \rightarrow \exp \left(\frac{1}{16}\left(a^{2}+2 a\right) \sum_{i} y_{i}^{2}+o(1)\right)
$$

as $n \rightarrow \infty, m / n \rightarrow$ a, uniformly over $\left(h_{1}, \ldots, h_{k}\right)$ belonging to compact domains of $(\mathbb{R} \backslash 0)^{k}$.

\section{Convergence to the GUE-corners process}

We now consider the joint distribution of the positions of the horizontal lozenges closest to the left flat boundary of the domain. Let $Y_{n, m}^{k}$ denote the $k$-tuple $Y_{n, m}^{k}=\left(y_{1}^{k}, y_{2}^{k}, \ldots, y_{k}^{k}\right)$, where $y^{k}=\left\{y_{j}^{k}\right\}$ are the positions of the horizontal lozenges on the $k$-th vertical line a uniformly random tiling $T_{n, m}^{f}$, as depicted in Figure 1. We show that the joint distribution of the ensemble $\left[Y_{n, m}^{1}, \ldots, Y_{n, m}^{k}\right]$, shifted by $m / 2$ and rescaled by a factor of $\sqrt{n}$, converges to the GUE-corners process.

In order to prove the convergence to GUE we use moment generating functions. Introduce the multivariate normalized Bessel function in $(x ; y)=\left(x_{1}, \ldots, x_{k} ; y_{1}, \ldots, y_{k}\right)$, defined as

$$
B_{k}(x ; y)=\frac{\operatorname{det}\left[\exp \left(x_{i} y_{i}\right)\right]_{i, j=1}^{k}}{\prod_{i<j}\left(x_{i}-x_{j}\right) \prod_{i<j}\left(y_{i}-y_{j}\right)} \prod_{i<j}(j-i)=\frac{s_{y-\delta_{k}}\left(e^{x}\right)}{s_{y-\delta_{k}}\left(1^{k}\right)} \frac{\Delta\left(e^{x}\right)}{\Delta(x)},
$$

where $\delta_{k}=(k-1, k-2, \ldots, 1,0)$ and $\Delta(z)=\prod_{i<j}\left(z_{i}-z_{j}\right)$ is the Vandermonde determinant.

Let $\mathbb{G U E}_{k}$ denote the $k$ eigenvalues $\varepsilon_{1} \geq \varepsilon_{2} \geq \cdots \geq \varepsilon_{k}$ of a random Hermitian $k \times k$ matrix from a Gaussian Unitary Ensemble. The idea is to show that the m.g.f. $\mathbb{E} B_{k}$ for the uniformly random lozenge tilings from $T_{f}(n, m)$ converges to the corresponding m.g.f. for $\mathbb{G U E}_{k}$, computed in [GP, Prop 5.6] as:

$$
\mathbb{E} B_{k}\left(x ; \mathbb{G} \mathbb{U} \mathbb{E}_{k}\right)=\exp \left(\frac{1}{2}\left(x_{1}^{2}+\cdots x_{k}^{2}\right)\right)
$$

Proposition 4.1 We have that the expectation of $B_{k}\left(x ; Y_{n, m}^{k}\right)$, where $Y_{n, m}^{k}$ is the $k$-tuple of the positions of the horizontal lozenges at line $k$ of a tiling chosen uniformly from $T_{n, m}^{f}$, is given by

$$
\mathbb{E} B_{k}\left(x ; \frac{Y_{n, m}^{k}-m / 2}{\sqrt{n}}\right)=\prod_{i=1}^{k} \exp \left(-\frac{m}{2 \sqrt{n}} x_{i}\right) \Phi_{m}\left(e^{x_{1} / \sqrt{n}}, \ldots, e^{x_{k} / \sqrt{n}} ; n\right) \frac{\Delta\left(e^{x / \sqrt{n}}\right)}{\Delta(x / \sqrt{n})} .
$$

Proof: First, we calculate the probability that $\left\{y_{j}^{k}\right\}$ are the positions of the horizontal lozenges on the $k$-th vertical line of a uniformly random tiling from $T_{n, m}^{f}$. Tilings in $T_{n, m}^{f}$ are in bijection with Gelfand-Tsetlin 
patterns of $n$ rows and entries no larger than $m$. Those, on the other hand, are in bijection with semistandard Young tableaux (SSYT) $T$ filled with the numbers $1, \ldots, n$ and shapes $\lambda$ (corresponding to the positions of the right-most horizontal lozenges as in Figure 1) that fit in the $m^{n}$ rectangle, i.e. $\lambda_{1} \leq m$. The positions of the horizontal lozenges on the $k$-th vertical line are simply the entries on the $k$-th row of the Gelfand-Tsetlin pattern. These correspond to the sub-tableaux of $T$ occupied by the entries which are no larger than $k$. See Figure 2 .

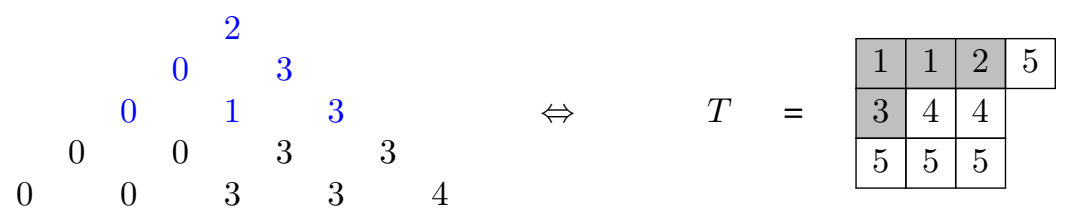

Fig. 2: The correspondence between the $k$-th line of a Gelfand-Tsetlin pattern and sub-tableau of an SSYT consisting of the entries $\leq k$. Here $\lambda=(4,3,3,0,0)$ and $y^{3}-\delta_{3}=(3,1,0)$.

The total number of tilings in $T_{n, m}^{f}$ is equal to the total number of SSYTs with entries $1, \ldots, n$ and shapes fitting inside $m^{n}$. The number of SSYTs with entries $1, \ldots, n$ of a given shape $\lambda$ is just $s_{\lambda}(\underbrace{1, \ldots, 1}_{n})$ and so the total number of tilings in $T_{n, m}^{f}$ is exactly $\phi_{m}(\underbrace{1, \ldots, 1}_{n})$. The number of tilings whose horizontal lozenges on the $k$-th diagonal are at positions $y^{k}=\left(y_{1}^{k}, \ldots, y_{k}^{k}\right)$, via the bijection with SSYTs described above, is equal to

$$
\sum_{\lambda \subset\left(m^{n}\right)} s_{y^{k}-\delta_{k}}(\underbrace{1, \ldots, 1}_{k}) s_{\lambda /\left(y^{k}-\delta_{k}\right)}(\underbrace{1, \ldots, 1}_{n-k})
$$

and thus the probability of the positions of the horizontal lozenges on the $k$-th vertical line being $y^{k}$ is just that last quantity divided by $\phi_{m}\left(1^{n}\right)$. Using the formula for $B_{k}$ as ratio of Schur functions, we get

$$
B_{k}\left(x ; \frac{y^{k}-\frac{m}{2}}{\sqrt{n}}\right) \frac{\Delta(x / \sqrt{n})}{\Delta\left(e^{x / \sqrt{n}}\right)}=\prod_{i} \exp \left(-\frac{x_{i} m}{2 \sqrt{n}}\right) s_{y^{k}-\delta_{k}}\left(E^{\frac{x_{1}}{\sqrt{n}}}, \ldots, e^{\frac{x_{k}}{\sqrt{n}}}\right) / s_{y^{k}-\delta_{k}}\left(1^{k}\right) .
$$

By the combinatorial description of Schur functions, for two alphabets $z, w$ we have that

$$
s_{\alpha}(z, w)=\sum_{\beta} s_{\beta}(z) s_{\alpha / \beta}(w),
$$

which we apply with $\alpha=\lambda, \beta=y^{k}-\delta_{k}, w=1^{n-k}$ and $z=e^{x / \sqrt{n}}$ :

$$
\begin{gathered}
\mathbb{E} B_{k}\left(x ; \frac{Y_{n, m}^{k}-m / 2}{\sqrt{n}}\right) \frac{\Delta(x / \sqrt{n})}{\Delta\left(e^{x / \sqrt{n}}\right)}=\sum_{y^{k}} B_{k}\left(x ; \frac{y^{k}-\frac{m}{2}}{\sqrt{n}}\right) \frac{\sum_{\lambda \subset\left(m^{n}\right)} s_{y^{k}-\delta_{k}}\left(1^{k}\right) s_{\lambda /\left(y^{k}-\delta_{k}\right)}\left(1^{n-k}\right)}{\phi_{m}\left(1^{n}\right)} \\
=\sum_{y^{k}} \prod_{i} \exp \left(-\frac{x_{i} m}{2 \sqrt{n}}\right) \frac{s_{y^{k}}\left(e^{\frac{x_{1}}{\sqrt{n}}}, \ldots, e^{\frac{x_{k}}{\sqrt{n}}}\right)}{s_{y^{k}-\delta_{k}}\left(1^{k}\right)} \frac{s_{y^{k}-\delta_{k}}\left(1^{k}\right) \sum_{\lambda \subset\left(m^{n}\right)} s_{\lambda /\left(y^{k}-\delta_{k}\right)}\left(1^{n-k}\right)}{\phi_{m}\left(1^{n}\right)}
\end{gathered}
$$




$$
=\prod_{i} \exp \left(-\frac{x_{i} m}{2 \sqrt{n}}\right) \frac{1}{\phi_{m}\left(1^{n}\right)} \sum_{\lambda \subset\left(m^{n}\right)} s_{\lambda}(e^{\frac{x_{1}}{\sqrt{n}}}, \ldots, e^{\frac{x_{k}}{\sqrt{n}}}, \underbrace{1, \ldots, 1}_{n-k})=\prod_{i} \exp \left(-\frac{x_{i} m}{2 \sqrt{n}}\right) \Phi_{m}\left(e^{\frac{x_{1}}{\sqrt{n}}}, \ldots, e^{\frac{x_{k}}{\sqrt{n}}} ; n\right) .
$$

Lemma 4.2 As $n, m \rightarrow \infty$ with $m / n \rightarrow a$, we have that

$$
\mathbb{E} B_{k}\left(x ; \frac{Y_{n, m}^{k}-m / 2}{\sqrt{n}}\right) \rightarrow \exp \left(1 / 16\left(a^{2}+2 a\right)\left(x_{1}^{2}+\cdots x_{k}^{2}\right)\right)
$$

Proof: This is a direct consequence of Proposition 4.1, the asymptotics of $\Phi_{m}$ from Theorem 3.4 and the fact that $\frac{\Delta(x / \sqrt{n})}{\Delta\left(e^{x / \sqrt{n}}\right)} \rightarrow 1$ uniformly on compact neighborhoods of $x$ as $n \rightarrow \infty$.

Theorem 4.3 We have that, as $n, m \rightarrow \infty$ with $m / n \rightarrow$ a for $a>0$,

$$
\frac{Y_{n, m}^{k}-m / 2}{\sqrt{n\left(a^{2}+2 a\right) / 8}} \rightarrow \mathbb{G U E}_{k}
$$

in the sense of weak convergence of random variables. Moreover, the so-rescaled positions of the horizontal lozenges on the first $k$ vertical lines, i.e. $\left\{\frac{Y_{n, m}^{j}-m / 2}{\sqrt{n\left(a^{2}+2 a\right) / 8}}\right\}_{j=1}^{k}$ weakly converge as random variables to the collection of eigenvalues $\left\{\varepsilon^{j}\right\}_{j=1}^{k}$ of the principle submatrices from a $k \times k$ matrix from the $\mathbb{G} \mathbb{U E}$ ensemble, where $\left\{\varepsilon^{j}\right\}$ are the eigenvalues of the submatrix formed by the first $j$ rows and columns.

Proof sketch:: It is a classical result, following Lévy's continuity theorem, that if the moment generating functions (MGF) $\mathbb{E}\left[e^{X_{i} t}\right], i=1, \ldots$ of a sequence of random variables $\left\{X_{i}\right\}_{i=1, \ldots}$ converge uniformly in a compact domain of $t$ to the MGF of a given random variable $X$, then $X_{i} \rightarrow X$ in distribution (i.e. weakly). This statement easily generalizes when replacing the random variables by vectors of random variables and MGF by $\mathbb{E} B_{k}$. This fact applies to the sequences $Y_{n, m}^{k}$ since

$$
\begin{aligned}
\mathbb{E} B_{k}\left(x ; \frac{Y_{n, m}^{k}-m / 2}{\sqrt{n\left(a^{2}+2 a\right) / 8}}\right)=\mathbb{E} B_{k}\left(\frac{x}{\sqrt{\left(a^{2}+2 a\right) / 8}} ; \frac{Y_{n, m}^{k}-m / 2}{\sqrt{n}}\right) \\
\rightarrow \exp \left(\frac{1}{2}\left(x_{1}^{2}+\cdots x_{k}^{2}\right)\right)=\mathbb{E} B_{k}\left(x ; \mathbb{G U E}_{k}\right),
\end{aligned}
$$

where $B_{k}(x ; y \alpha)=B_{k}(\alpha x ; y)$ for any constant $\alpha$ and Lemma 4.2 gives the asymptotics.

The convergence of the entire collection of horizontal positions $\left\{\frac{Y_{n, m}^{j}-m / 2}{\sqrt{n\left(a^{2}+2 a\right) / 8}}\right\}_{j=1}^{k}$ to the collection of eigenvalues of all the principal submatrices follows from the Gibbs property satisfied by both collections: given the vector $Y_{n, m}^{k}$, the distribution of the horizontal lozenges on the first $k-1$ vertical lines is clearly uniform subject to the interlacing conditions; the same holds for the eigenvalues $\left\{\varepsilon^{j}\right\}_{j=1}^{k-1}$ given $\varepsilon^{k}$, which are again subject to the interlacing conditions, i.e. $\varepsilon_{i-1}^{j} \geq \varepsilon_{i-1}^{j-1} \geq \varepsilon_{i}^{j}$ (see [Baryshnikov]).

We can now compare the results for the free boundary case and the hexagon by comparing with the results for the hexagon implicit or explict in [GP] and [Johansson-Nordenstam, Nordenstam, Novak], which proves the first claim of the Main Theorem. 


\section{Limit shape}

We now show the existence of a limit shape for the height function (alternatively, the symmetric plane partition) of the tilings with free boundary, which does not follow immediately from other previously considered cases.

We prove that the positions of the horizontal lozenges at any vertical line $x=\alpha n$, given by a signature (partition) $\mu^{\alpha n}$, converge to a "limit shape" in the following sense. This means that there is a monotone piecewise continuous function $f_{\alpha}(t):[0, b] \rightarrow \mathbb{R}$ for some sufficiently large $b$, such that

$$
\lim _{n \rightarrow \infty} \frac{\mu_{i}^{\alpha n}+\alpha n-i}{\alpha n} \rightarrow f\left(\frac{i}{\alpha n}\right)
$$

in probability (see e.g. [KOS, Section 6.2]), where $\mu^{\alpha n}$ is now a random signature of length $\alpha n$, according to some probability measure on signatures. Moreover, the "limit shape" of the random tilings as $n \rightarrow \infty$ should be a function $H: \mathbb{R}^{2} \rightarrow \mathbb{R}$. At a point $(\alpha, t)$, this is $H(\alpha, t)=f_{\alpha}(t)$. For any signature $\lambda$ of length $N$, define the the counting measure

$$
m[\lambda]=\frac{1}{n} \sum_{i=1}^{N} \delta\left(\frac{\lambda_{i}+N-i}{N}\right),
$$

where $\delta$ is the Dirac delta measure. Clearly, $m[\lambda]$ is a compactly supported probability measure.

As shown in [BBO, Proposition 2.2], if we prove that the sequence of random measures $\mu^{N}$ converge to a deterministic probability measure $m$, this automatically implies the convergence of the limit shape, which is then simply the distribution function of $m$. Following ideas from [BG] we now prove such convergence.

For any real $\alpha \in(0,1)$ and signature $\lambda$ of $n$ parts, define for any signature $\mu=\left(\mu_{1} \geq \cdots \geq \mu_{\lfloor\alpha n\rfloor}\right)$ of $\lfloor\alpha n\rfloor$ parts, the following probability

$$
P_{m, n}^{\alpha}(\mu)=\frac{\sum_{\lambda: \lambda_{1} \leq m} s_{\lambda / \mu}\left(1^{n-\lfloor\alpha n\rfloor}\right) s_{\mu}\left(1^{\lfloor\alpha n\rfloor}\right)}{\sum_{\lambda: \lambda_{1} \leq m} s_{\lambda}\left(1^{n}\right)} .
$$

Combinatorially this is the probability that a random SSYT with at most $m$ columns and $N$ letters will have the first $\lfloor\alpha n\rfloor$ letters forming an SSYT of shape $\mu$. Thus, it is evident that summing over all possible shapes $\mu$, we obtain all SSYT of shape $\lambda$ and $n$ letters, and thus $P^{\alpha, \lambda}$ is a probability measure on the signatures of length $\lfloor\alpha n\rfloor$.

Finally, for any probability measure $\rho$ on the set of signatures of length $N$, define the $G L_{N}$-character generating function

$$
S_{\rho}\left(u_{1}, \ldots, u_{N}\right)=\sum_{\mu: \ell(\mu)=N} \rho(\mu) \frac{s_{\mu}\left(u_{1}, \ldots, u_{N}\right)}{s_{\mu}\left(1^{N}\right)} .
$$

Given a probability distribution $\rho$ on signatures $\lambda$, we denote (by a slight abuse of notation, to agree with $[\overline{\mathrm{BG}}])$ by $m[\rho]$ the random counting measures $m[\lambda]$, where $\lambda \sim \rho$.

We will apply the following result, proven in $[\overline{\mathrm{BG}}]$ and inspired by $[\overline{\mathrm{BBO}}]$. 
Theorem 5.1 (Theorem 5.1 in $\left[\overline{\mathbf{B G}])}\right.$ For each $N$, let $\rho^{N}$ be a measure on the set of signatures of length $N$. Suppose that for every $k$

$$
\lim _{N \rightarrow \infty} \frac{1}{N} \ln \left(S_{\rho^{N}}\left(u_{1}, \ldots, u_{k}, 1^{N-k}\right)\right)=Q\left(u_{1}\right)+\cdots+Q\left(u_{k}\right),
$$

where $Q$ is an analytic function in a neighborhood of 1 and the convergence is uniform in an open (complex) neighborhood of $(1, \ldots, 1)$. Then the random pushforward measures $m\left[\rho^{N}\right]$ converge, as $N \rightarrow \infty$, in probability, in the sense of moments, to a deterministic measure $M$ on $\mathbb{R}$, whose moments are given by

$$
\int_{\mathbb{R}} x^{r} M(d x)=\left.\sum_{\ell=0}^{r}\left(\begin{array}{l}
r \\
\ell
\end{array}\right) \frac{1}{(\ell+1) !} \frac{\partial^{\ell}}{\partial u^{\ell}} Q(u)\right|_{u=1}
$$

We apply this theorem to the measure $\rho=P_{m, n}^{\alpha}$. This measure is the distribution of the partition $\mu$, which represents the positions (height function) of the horizontal lozenges at the vertical line $k=\alpha n$, i.e. $y^{k}$ as in Section 4 (see Figure 1), of a uniformly random lozenge tiling from $T_{f}(n, m)$. We have that $N=\lfloor\alpha n\rfloor$, and then compute as in Section 4

$$
\begin{aligned}
& S_{P_{m, n}^{\alpha}}\left(u_{1}, \ldots, u_{N}\right)=\sum_{\mu: \ell(m u)=N} P_{m, n}^{\alpha}(\mu) \frac{s_{\mu}\left(u_{1}, \ldots, u_{N}\right)}{s_{\mu}\left(1^{N}\right)} \\
& \quad=\sum_{\mu: \ell(\mu)=N} \frac{\sum_{\lambda: \lambda_{1} \leq m} s_{\lambda / \mu}\left(1^{n-N}\right) s_{\mu}\left(1^{N}\right)}{\sum_{\lambda: \lambda_{1} \leq m} s_{\lambda}\left(1^{n}\right)} \frac{s_{\mu}\left(u_{1}, \ldots, u_{N}\right)}{s_{\mu}\left(1^{N}\right)}=\Phi_{m}\left(u_{1}, \ldots, u_{N} ; n\right)
\end{aligned}
$$

Proposition 5.2 Let $m, n \rightarrow \infty$ with $m / n \rightarrow a$, where $a$ is a positive real number. Then for any fixed $k$, we have

$$
\lim _{n \rightarrow \infty} \frac{1}{n} \ln \Phi_{m}\left(u_{1}, \ldots, u_{k} ; n\right)=\Psi_{a}\left(u_{1}\right)+\cdots+\Psi_{a}\left(u_{k}\right),
$$

where $\Psi_{a}\left(e^{y}\right)=y \frac{a}{2}+2 \phi(y ; a)-2$ and $\phi$ is defined as follows

$$
\begin{aligned}
h(y)=\frac{1}{4}\left(\left(e^{y}+1\right)\right. & \left.+\sqrt{\left(e^{y}+1\right)^{2}+\left(a^{2}+2 a\right)\left(e^{y}-1\right)^{2}}\right) \\
\phi(y ; a)=\left(\frac{a}{4}+1\right) & \ln \left(h(y)-\left(\frac{a}{4}+1\right)\left(e^{y}-1\right)\right)-\left(\frac{a}{4}+\frac{1}{2}\right) \ln \left(h(y)-\left(\frac{a}{4}+\frac{1}{2}\right)\left(e^{y}-1\right)\right) \\
& +\frac{a}{4} \ln \left(h(y)+\frac{a}{4}\left(e^{y}-1\right)\right)-\left(\frac{a}{4}-\frac{1}{2}\right) \ln \left(h(y)+\left(\frac{a}{4}-\frac{1}{2}\right)\left(e^{y}-1\right)\right)
\end{aligned}
$$

Proof sketch: Using the formula for $\Phi$ in terms of $\mathfrak{X}$, we find the asymptotics for $\mathfrak{X}$ in the given regime. Using the relationship to $S$, we first compute $\lim _{N \rightarrow \infty} \frac{\ln S_{\nu^{m}}\left(e^{y} ; N\right)}{N}=\phi(y ; a)-1$. Since both sides are analytic around $y=0$, the equality extends to a neighborhood of 0 . In the case of $m=0$, i.e. for $f_{0}$ we calculate directly using the same methods (also justified in [GP, Example 1]) $\lim _{N \rightarrow \infty} \frac{\ln S_{\nu^{0}}\left(e^{y} ; N\right)}{N}=0$. By Prosition 2.1. the same limits hold when replacing $S_{\nu}$ by the corresponding $\mathfrak{X}_{\tau}$ for one variable. Proposition 3.3. we obtain the corresponding limit for the multivariate characters $\mathfrak{X}_{\tau^{m}}$ and $\mathfrak{X}_{\tau^{0}}$. 
Theorem 5.3 Let $n, m \in \mathbb{Z}$, such that $m / n \rightarrow$ a as $n \rightarrow \infty$, where $a \in(0,+\infty)$. Let $H(u, v)$ be the height function of the uniformly random lozenge tiling in $T_{f}(n, m)$ (alternatively, the value of the square in the corresponding plane partition at position $(v, u-v)$ with $(0,0)$ at the lower left corner). Then as $n \rightarrow \infty$, for all $x \in(0,1), y \geq 0$ the normalized $\frac{1}{n} H(n x, n y)$ converges in probability to a deterministic function $L(x, y)$, referred to as "the limit shape".

Moreover, for any fixed $x \in(0,1)$, the function $L(x, y)$ is the distribution function of the limit measure $M$ whose moments are given by

$$
\int_{\mathbb{R}} x^{r} M(d x)=\left.\sum_{\ell=0}^{r}\left(\begin{array}{l}
r \\
\ell
\end{array}\right) \frac{1}{(\ell+1) !} \frac{\partial^{\ell}}{\partial u^{\ell}} \Psi_{a}(u)\right|_{u=1},
$$

where $\Psi_{a}(u)$ is defined in Proposition 5.2

Proof: The distribution $\rho^{n}(\mu)=P_{m, n}^{\alpha}(\mu)$ defined in equation (6) is a probability distribution on the set of signatures of length $\lfloor\alpha n\rfloor$, as explained there. Further, the corresponding $G L_{n}$-character generating function $S_{\rho^{n}}$ is, by equation (7), equal to $\Phi_{m}$. By Proposition 5.2, this $S_{\rho^{n}}$ satisfies the conditions of Theorem 5.1] so the random measures $m[\mu]$, defined by the random signatures $\mu$ distributed according to $P_{m, n}^{\alpha}$, converge in probability to a deterministic measure $M$, defined accordingly through its moments. Finally, Theorem 5.3 applies to this measure $M$ and we obtain the desired limit of the height function $L(x, y)$, which is given as the distribution function of $M$.

To show the Main Theorem, we can compare this result to the analogous statement for the full hexagon: either by the same methods show that it's deterministic measure $M$ has the same moments or compare with the present results from $[\mathrm{BG}, \mathrm{CLP}, \mathrm{CKP}]$.

\section{Asymptotics II: when $\lim _{n \rightarrow \infty} \frac{m}{n}=0, \infty$}

So far, for reasons concerning the physics nature of the models, the interest has been in studying the "scaling limits" of lattice models, in which the scaling factors are the same in all directions. Here we consider other regimes, in which the scalings in the different directions differ in growth order. For example, the vertical scaling could be proportional to $m \sim \sqrt{n}$ and the horizontal is $n$. We consider again the positions of the horizontal lozenges near the boundary, where now it is not a priori obvious what their correct scaling should be, in order to get a limiting distribution for $Y_{n, m}^{k}$ as $m, n \rightarrow \infty$. The following propositions are complementary to Theorem 4.3

Proposition 6.1 Suppose that $m, n \rightarrow \infty$ with $m / n \rightarrow \infty$, and suppose that $\frac{n^{2}}{m}=o(1)$. Then the shifted rescaled positions $\left(Y_{n, m}^{k}\right)$ of the horizontal lozenges on the kth vertical line satisfy:

$$
\frac{Y_{n, m}^{k}-m / 2}{m / \sqrt{8 n}} \rightarrow \mathbb{G U E}_{k}
$$

in distribution for all fixed $k$. The collection $\left\{\frac{Y_{n, m}^{j}-m / 2}{m / \sqrt{8 n}}\right\}_{j=1}^{k}$ converges to the GUE-corners process. 
Proposition 6.2 Let $n, m \rightarrow \infty$, such that $n / m \rightarrow \infty$. Then

$$
\frac{Y_{n, m}^{k}-m / 2}{2 \sqrt{m}} \rightarrow \mathbb{G U E}_{k}
$$

and the collection of shifted rescaled positions of the horizontal lozenges on lines $1, \ldots, k$ converges to the $k \times k$ GUE-corners process.

\section{Acknowledgements}

We would like to thank Philippe Di Francesco for asking the author about GUE in the free boundary tilings. We also thank Alexei Borodin, Vadim Gorin, Christian Krattenthaler and Nicolai Reshetikhin for helpful discussions on further problems and the background literature. The author was partially supported by a Simons postdoctoral fellowship while doing this work at UCLA.

\section{References}

[BBO] A. Borodin, A. Bufetov, G. Olshanski, Limit shapes for growing extreme characters of $U(\infty)$. arXiv:1311.5697.

[BG] A. Bufetov, V. Gorin, Representations of classical Lie groups and quantized free convolution, arXiv: 1311.5780 .

[CKP] H. Cohn, R. Kenyon, J. Propp, A variational principle for domino tilings. J. Amer. Math. Soc. 14 (2001), no. 2, 297-346. arXiv:math/0008220.

[CLP] H. Cohn, M. Larsen, J. Propp, The Shape of a Typical Boxed Plane Partition, New York J. Math., 4(1998), 137-165. arXiv: math/9801059.

[DR] Ph. Di Francesco, N. Reshetikhin, Asymptotic shapes with free boundaries. Comm. Math. Phys. 309 (2012), no. 1, 87-121.

[GP] V. Gorin, G. Panova, Asymptotics of symmetric polynomials with applications to statistical mechanics and representation theory, Annals of Probability, to appear (2014).

[KO] R. Kenyon, A. Okounkov, Limit shapes and Burgers equation, Acta Math. 199 (2007), no. 2, 263-302. arXiv:math-ph/0507007.

[KOS] R. Kenyon, A. Okounkov, S. Sheffield, Dimers and amoebae. Ann. of Math. (2) 163 (2006), no. 3, 10191056 .

[Mac] I. G. Macdonald, Symmetric functions and Hall polynomials, Second Edition. Oxford University Press, 1999.

[OR] A. Yu. Okounkov, N. Yu. Reshetikhin, The birth of a random matrix, Mosc. Math. J., 6, no. 3 (2006), 553-566.

[EC2] R. P. Stanley, Enumerative combinatorics Vol. 2. Cambridge Studies in Advanced Mathematics, 62. Cambridge University Press, Cambridge, 1999. 